\title{
Alteration of macrophage immune phenotype in a murine sepsis model is associated with susceptibility to secondary fungal infection
}

\author{
Chau Tran Bao Vu, ${ }^{1}$ Arsa Thammahong, ${ }^{2}$ Asada Leelahavanichkul, ${ }^{3,4}$ Patcharee Ritprajak ${ }^{5,6}$
}

\begin{abstract}
Background: Secondary fungal infection is a major complication in patients with sepsis-associated immunosuppression. However, sepsis-induced immune alterations related to fungal susceptibility have not been well characterized.

Objective: To determine kinetic changes in the immune phenotype by determining the proportion of T cells, B cells and macrophages, and especially the expression of an immune exhaustion marker PD-1, in murine sepsis. In addition, sepsis-induced alterations of these immune cells were assessed in relation to susceptibility to secondary fungal infection.
\end{abstract}

Methods: Cecal ligation and puncture (CLP) was used as a mouse sepsis model, with Candida albicans as the secondary systemic fungal infection. Splenic T cells, B cells and macrophages were assessed by flow cytometry.

Results: Alterations in T cell and B cell numbers and the proportion of PD-1 expressing T cells and B cells in CLP mice were not clearly related to susceptibility to secondary Candida infection. By contrast, changes in levels of CD86 ${ }^{+}-$ activated macrophages, and the proportion of the $\mathrm{PD}-1^{+}$population among the $\mathrm{CD} 86^{+}$macrophages in CLP mice were found to be related to secondary fungal infection susceptibility.

Conclusion: Macrophage activation and exhaustion might be a significant determinant in susceptibility to fungal infection, and outcomes of infection. This study provided more comprehensive knowledge pertinent to patient evaluation and therapeutics design in restoring host defenses against secondary fungal infection in those with sepsis.

Key words: macrophage, immune phenotype, sepsis, CD86, PD-1

\section{Citation:}

Vu, C. T. B., Thammahong, A., Leelahavanichkul, A., Ritprajak, P. (2022). Alteration of macrophage immune phenotype in a murine sepsis model is associated with susceptibility to secondary fungal infection. Asian Pac J Allergy Immunol, 40(2), 163-172. https://doi.org/10.12932/ap-170519-0565

\section{Affiliations:}

${ }^{1}$ Graduate Program in Oral Biology, Faculty of Dentistry,

Chulalongkorn University, Bangkok, Thailand

2 Antimicrobial Resistance and Stewardship Research Unit, Department of Microbiology, Faculty of Medicine, Chulalongkorn University, Bangkok, Thailand

${ }^{3}$ Immunology Unit, Department of Microbiology, Faculty of Medicine, Chulalongkorn University, Bangkok, Thailand
${ }^{4}$ Translational Research in Inflammation and Immunology Research Unit (TRIRU), Department of Microbiology, Chulalongkorn University, Bangkok, Thailand

${ }^{5}$ Department of Microbiology, Faculty of Dentistry, Chulalongkorn University, Bangkok, Thailand

${ }^{6}$ Research Unit in Integrative Immuno-Microbial Biochemistry and Bioresponsive Nanomaterials, Department of Microbiology, Chulalongkorn University, Bangkok, Thailand

\section{Corresponding author:}

1. Patcharee Ritprajak

Department of Microbiology, Faculty of Dentistry, Chulalongkorn University, Bangkok 10330, Thailand E-mail address: Patcharee.R@chula.ac.th

2. Asada Leelahavanichkul Immunology Unit, Department of Microbiology, Faculty of Medicine Chulalongkorn University, Bangkok 10330, Thailand E-mail: aleelahavanit@gmail.com 


\section{Introduction}

Sepsis, a life-threatening condition caused by severe systemic infection, is one of the most common causes of death in critically ill patients. ${ }^{1,2}$ Immune responses in patients with sepsis consist of a cytokine-mediated hyper-inflammatory phase, which most patients survive, and a subsequent immunosuppressive phase (or immune paralysis) where immune cells are exhausted and dysfunctional. These two immune events in sepsis may occur simultaneously or sequentially. ${ }^{1}$

Fungal infections have emerged as a problem over the last few decades in relation to an increased incidence of anti-fungal drug resistance. In addition, the immune status in patients is a crucial determinant for susceptibility to and outcome of fungal infections. Secondary fungal infections are a main cause of nosocomial infections worldwide, leading to high morbidity and mortality. ${ }^{3,4}$ Patients with sepsis-associated immunosuppression have increased susceptibility to secondary fungal infections, such as invasive candidiasis and aspergillosis. ${ }^{5,6}$ As an opportunistic pathogenic fungus, Candida albicans remains the leading cause of nosocomial bloodstream infections. The mortality rate of candidemia in intensive care patients is high, being approximately $50 \%$ without septic shock and $90 \%$ with septic shock. ${ }^{7}$

Programmed cell death-1 (PD-1 or CD279) is an immune checkpoint molecule, which triggers an inhibitory signal that modulates immune cell activation and function. ${ }^{8}$ Persistent upregulation of PD-1 on T cells in chronic viral and bacterial infections is associated with T-cell exhaustion. ${ }^{9}$ Substantial PD-1 expression on splenocytes and on circulating immune cells, including monocytes and $\mathrm{T}$ cells, has been observed in patients with severe sepsis. ${ }^{10,11}$ In addition, mice with cecal ligation and puncture (CLP)-induced sepsis displayed high PD-1 expression on splenic $\mathrm{T}$ cells, while blockade of the PD-1 pathway attenuated sepsis severity. ${ }^{12,13}$

A previous study investigated the expression of $\mathrm{PD}-1$ on $\mathrm{T}$ cells, B cells and CD11 b cells in a CLP model, and examined the role of $\mathrm{PD}-1$ in sepsis by pre-treatment with anti-PD-1 monoclonal antibody. ${ }^{13}$ However, the observation of PD-1 expression was limited to day 1 post-sepsis, and the authors only determined the role of PD-1 in the context of primary sepsis. In this current study, kinetic changes in the proportion of T cells, B cells and macrophages were examined, along with PD-1 expression by these cells, and their association with secondary fungal infection after sepsis, since this was a major complication in septic patients with immune paralysis. ${ }^{1,11}$ C. albicans was used as the secondary fungal infection in the mouse model because it was a leading cause of opportunistic infection worldwide, and had been widely used as an experimental model of fungal infection. The findings provided more comprehensive knowledge of sepsis-induced immune alterations and their relation to secondary infection, which could be used for patient evaluation and the selection of therapeutic approaches.

\section{Methods \\ Animals and the animal model}

All animal procedures were conducted according to guidelines of the National Institutes of Health (NIH), USA, and were approved by the Institutional Animal Care and Use Committee (IACUAC) of the Faculty of Medicine, Chulalongkorn University, Bangkok, Thailand (protocol number 031/2561). Six to eight-week-old female C57BL/6 mice were purchased from the National Laboratory Animal Center of Mahidol University, Bangkok, Thailand, and were housed in the Animal Facility Center of the Medical School, Chulalongkorn University.

CLP was performed under isoflurane anesthesia as described previously. ${ }^{14}$ Briefly, one centimeter from the cecal tip, the cecum was ligated and punctured with a 21-guage needle. For the control mice, the cecum was exteriorized, but neither ligated nor punctured. Primaxin IV (Merck, Kenilworth, NJ, USA) at $10 \mathrm{mg} / \mathrm{kg}$ in $100 \mu \mathrm{L}$ normal saline (NSS) was subcutaneously administered immediately after CLP, and once daily thereafter. For post-operative analgesia, $10 \mathrm{mg} / \mathrm{kg}$ tramadol (Madhya Pradesh, India) in $100 \mu \mathrm{L}$ NSS was subcutaneously administered immediately after the operation, and at $6 \mathrm{~h}$ and 24 h later.

The CLP and control mice were secondary infected with C. albicans SC5314 (or ATCC MYA-2876). The yeast was cultured on yeast extract peptone dextrose (YPD) agar (HiMedia, Mumbai, India) at $30^{\circ} \mathrm{C}$ for $48 \mathrm{~h}$, and was then harvested and washed with phosphate-buffered saline (PBS). Yeast cells at 2 $\times 10^{5}$ cells in $100 \mu \mathrm{L}$ NSS were intravenously administered via a tail vein at day 1 , day 5 and day 12 post-CLP, under isoflurane anesthesia.

\section{Blood analysis of mice}

Renal and liver injury was assessed by the level of serum creatinine and alanine transaminase (ALT), using QuantiChrom Creatinine and EnzyChrom Alanine Transaminase assay kits (BioAssay Systems, Hayward, CA, USA), respectively. The detection of serum cytokines was performed with enzyme-linked immunosorbent assay (ELISA) kits (Biolegend, San Diego, CA, USA). To determine blood fungal burden, different volumes of blood were plated onto Sabouraud dextrose agar (SDA) containing $0.1 \%$ chloramphenicol (Thermo Scientific, Basingstoke, UK), and were then incubated at $35^{\circ} \mathrm{C}$, for $72 \mathrm{~h}$ before fungal colony counting.

\section{Flow cytometry of splenocytes}

Fluorochrome-conjugated antibodies were obtained from Biolegend (San Diego, CA, USA): anti-mouse F4/80 (BM8), anti-mouse CD86 (GL1), anti-mouse MHC class II (M5/114.15.2), anti-mouse PD1 (29F.1A12), anti-mouse CD4 (RM4-5), anti-mouse CD8 (53-6.7), and anti-mouse B220 (RA3-6B2). Splenocytes were prepared, stained with the fluorochrome-conjugated antibodies and processed for flow cytometry on a CytoFLEX system according to the manufacturer's protocols, and data were analyzed with Kaluza software (Beckman Coulter, Brea, CA, USA). 
Total numbers of splenocytes were counted. The number of each type of immune cell was then calculated by multiplying its percentage from the flow cytometry analysis with the total numbers of splenocytes.

\section{Statistical analysis}

SPSS Statistics for Windows version 23.0 (IBM Corp., Armonk, NY, USA) and Prism 7.0 (GraphPad, San Diego, CA, USA) were used for statistical analysis. Data were presented as mean \pm standard error (SE). Student's $t$-test and One-way ANOVA with Bonferroni's post-hoc analysis were used to compare the differences between two groups and more than two groups, respectively. The log-rank test was used for survival analysis. Significant differences were accepted at $p<$ 0.05 .

\section{Results}

\section{Innate and adaptive immune response phase in the CLP mouse model}

To determine the phase of the immune response following sepsis, serum parameters were determined on day 1, 5 and 12 post-CLP. On day 1 post-CLP, serum creatinine and ALT were highly increased in CLP mice, indicating acute renal and liver injury, respectively (Figure 1A-B). Consistent with these tissue injuries, innate pro-inflammatory cytokines, TNF- $\alpha$ and IL-6, were markedly upregulated in serum of CLP mice, which defined a hyper-inflammatory state (Figure 1C-D).
Increased serum IL-10 production was also observed on day 1 post-CLP (Figure 1E). Serum creatinine, ALT, TNF- $\alpha$, IL6 , and IL-10 declined to baseline levels (equal to those of the control) from day 5 to 12 post-CLP (Figure 1A-E). Conversely, IFN- $\gamma$ was notably increased at day 5 , and partially decreased at day 12 post-CLP (Figure 1F). The high upregulation of organ injury markers and pro-inflammatory cytokines at an early phase of sepsis (day 1 post-CLP) might indicate a hyper-inflammatory state. By contrast, the decline in these markers and pro-inflammatory cytokines, in concert with the upregulation of the protective cytokine IFN- $\gamma,{ }^{15}$ suggested a protective or recovery state of sepsis.

\section{Alteration of $T$ cell, $B$ cell and macrophage numbers during sepsis}

The numbers of immune cells during sepsis were determined, and compared with the immune phases that were identified in Figure 1. CD4 T cells, CD8 T cells, and B cells were identified as $\mathrm{CD}^{+} \mathrm{CD}^{+}, \mathrm{CD}^{+} \mathrm{CD}^{+}$, and $\mathrm{B} 220^{+}$cells, respectively (Figure $2 \mathrm{~A}$ ). In CLP mice, total splenocyte numbers were reduced at day 1 , restored by day 5 , and significantly increased on day 12 post-CLP (Figure 2B). At day 1 post-CLP, CD4 T cell, CD8 T cell, and B cell numbers in CLP splenocytes were decreased in accordance with total splenocyte numbers (Figure 2C-H). Loss of CD4 T CD8 T and B cells in CLP splenocytes was further observed at day 5 and day 12 post-CLP (Figure $\mathbf{2 C - H}$ ), contrary to increased total splenocyte numbers (Figure 2A).
A
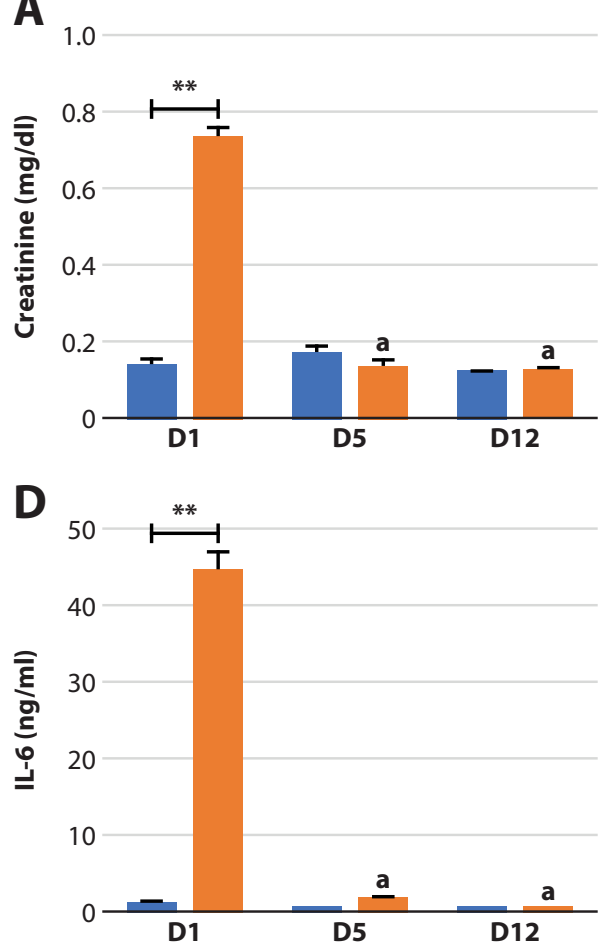

B

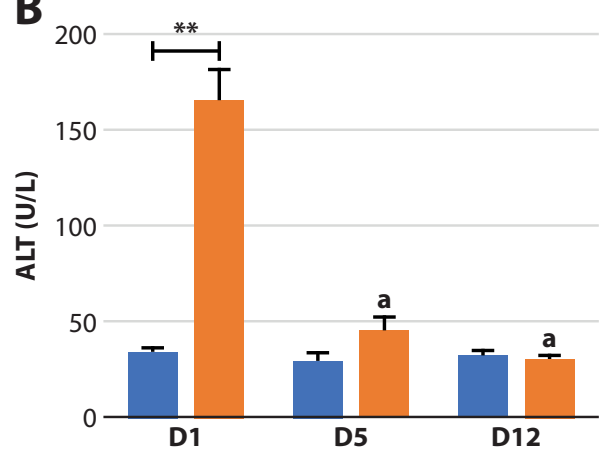

E

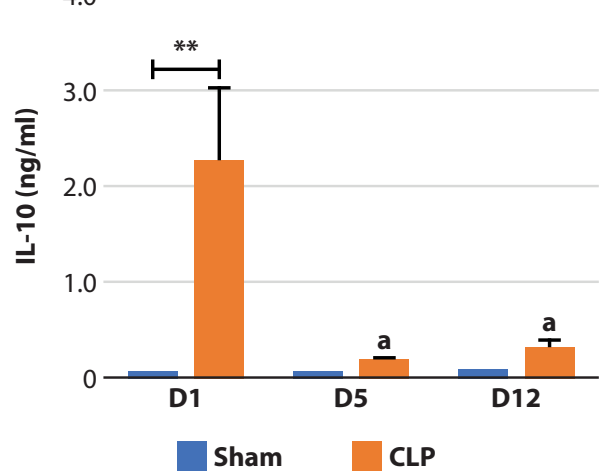

$C_{200}$

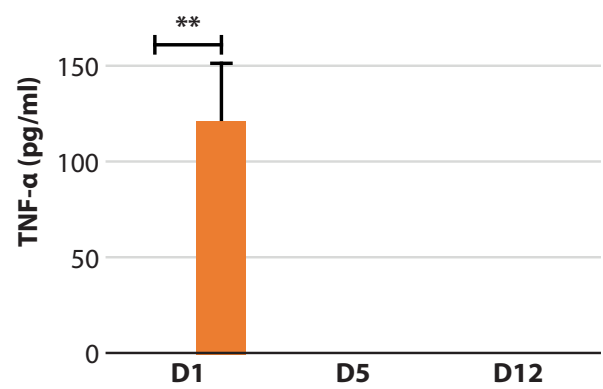

$\mathbf{F}$

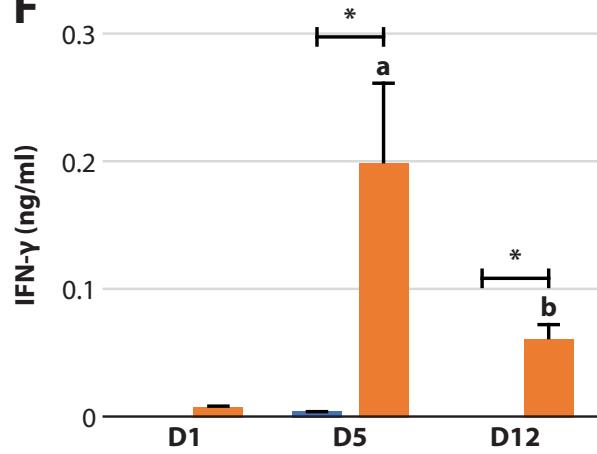

Figure 1. Kinetic changes of renal failure, liver injury and pro-inflammatory cytokines in CLP mouse model.

(A) Serum creatinine, (B) serum alanine transaminase (ALT), and (C-F) serum cytokines of CLP or sham control mice were determined at day 1 (D1), day 5 (D5) and day 12 (D12) post-CLP. $n=4 /$ group/time point. Data represent two independent experiments. ${ }^{\star} p<0.05 ;{ }^{* *} p<0.01 ;{ }^{\mathrm{a}} p<0.05$ vs. D1; ${ }^{\mathrm{b}} p<0.05$ vs. D5. 
A

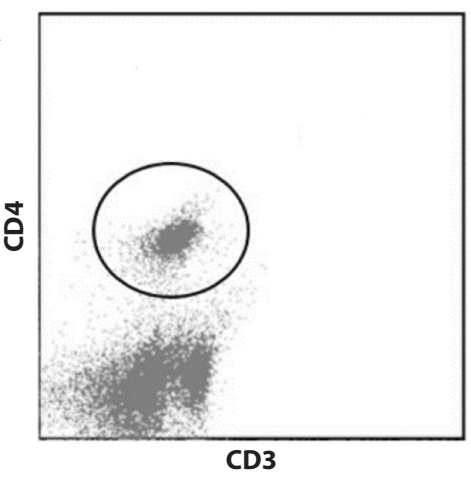

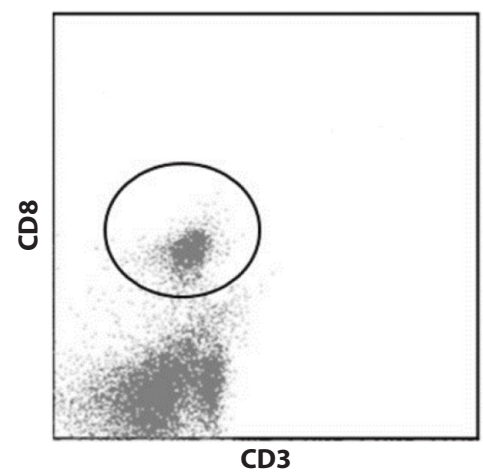

CD3

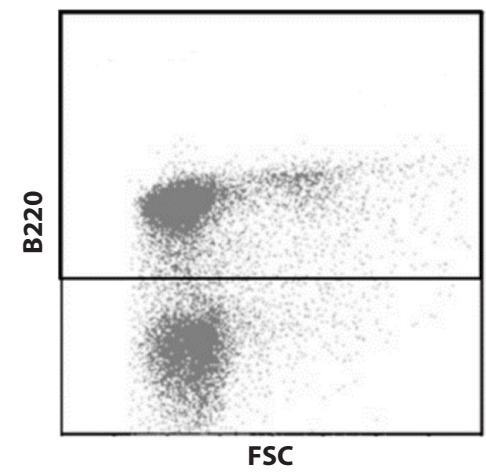

$\mathbf{D}^{\left(\times 10^{6}\right)}$

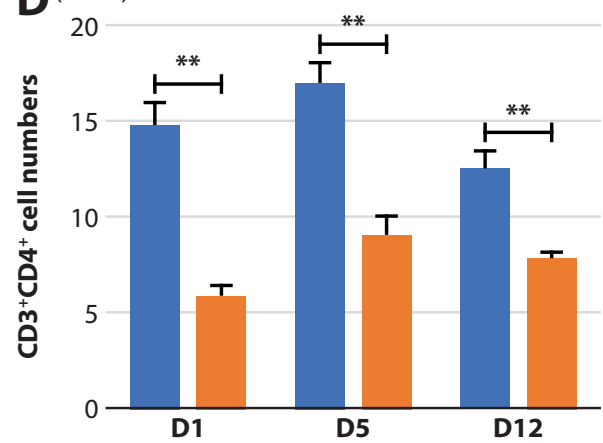

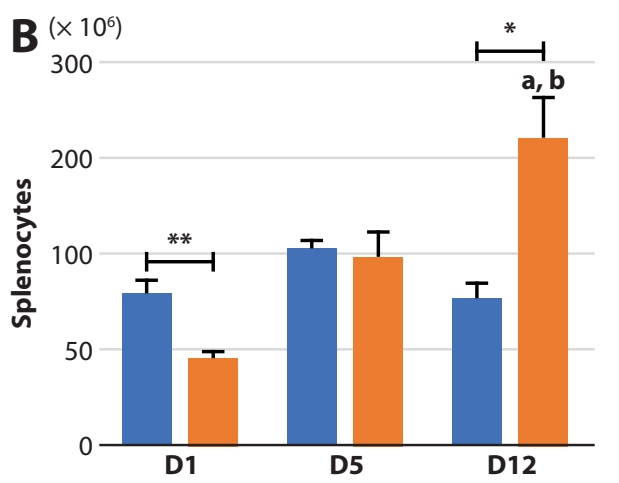

$C_{25}$
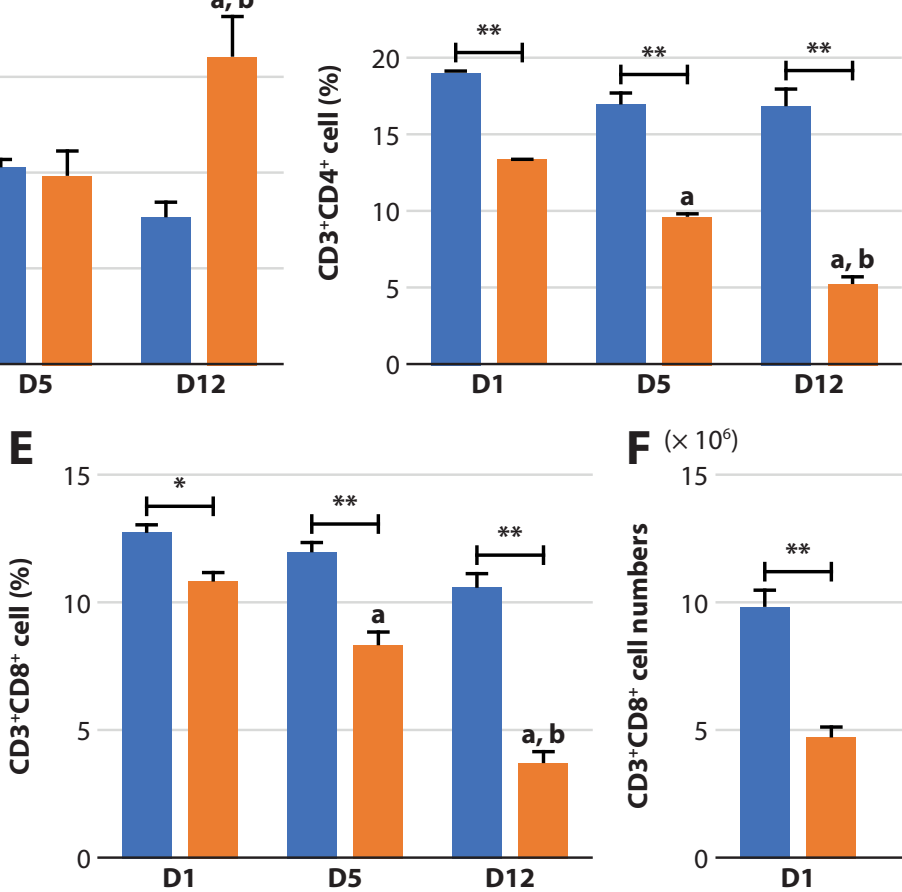

$\mathbf{F}\left(\times 10^{6}\right)$

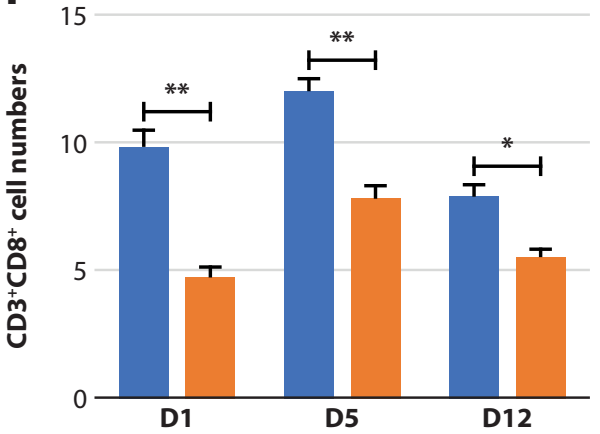

G

$\mathbf{H}^{\left(\times 10^{6}\right)}$
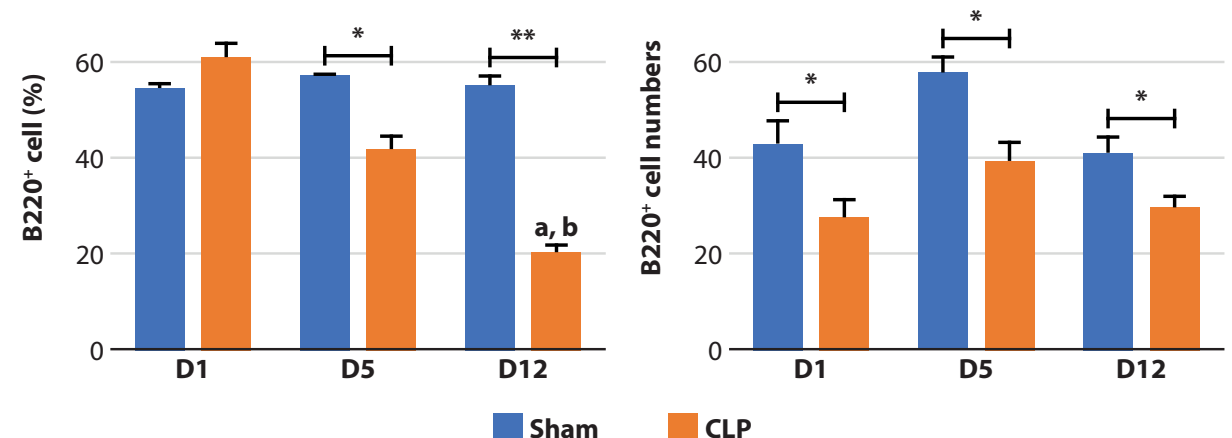

CLP

Figure 2. Alteration of $\mathrm{T}$ cell and $\mathrm{B}$ cell numbers during sepsis.

(A) Dot plot analysis of CD4 T cells $\left(\mathrm{CD}^{+}{ }^{+} \mathrm{CD} 4^{+}\right.$cells), CD8 T cells $\left(\mathrm{CD} 3^{+} \mathrm{CD} 8^{+}\right.$cells) and B cells (B220 ${ }^{+}$cells), (B) Total splenocyte numbers, (C) percentage of splenic CD4 T cells, (D) numbers of splenic CD4 T cells, (E) percentage of splenic CD8 T cells, (F) numbers of splenic CD8 T cell, $(\mathrm{G})$ percentage of splenic B cells, and $(\mathrm{H})$ numbers of splenic B cell of CLP and sham control mice were assessed at day 1 (D1), day 5 (D5) and day 12 (D12) post-CLP. $n=5 /$ group/time point. Data represent two independent experiments. ${ }^{\star} p<0.05 ;{ }^{*} p<0.01 ;{ }^{\mathrm{a}} p<0.05$ vs. D1; ${ }^{\mathrm{b}} p<0.05$ vs. D5. 
As an innate immune cell, macrophages played multiple roles in sepsis, with extensive effects on inflammatory and homeostatic processes. ${ }^{16,17}$ For this reason, we determined macrophage numbers and activation. Macrophages were identified as $\mathrm{F} 4 / 80^{+}$cells, and their activation state was determined based on the expression of CD86 and MHC class II (Figure $3 \mathbf{A})$. At day 1 post-CLP, the percentage of macrophages (F4/80+ cells) in spleen of CLP mice was comparable to that of the control (Figure 3B); however, macrophage numbers were significantly decreased (Figure 3C). Opposing the reduction in adaptive cell numbers, macrophages in CLP splenocytes were slightly increased at day 5 post-CLP, and substantial numbers of macrophages were detected at day 12 post-CLP
(Figure 3C), consistent with elevated splenocyte numbers (Figure 2B). The percentage of $\mathrm{F} 4 / 80^{+} \mathrm{MHC}$ class $\mathrm{II}^{+}$cells was decreased on days 5 and 12 post-CLP (Figure 3D). Notably, the proportion of $\mathrm{CD} 6^{+} \mathrm{F} 480^{+}$cells was reduced at day 5; however, it was greatly increased on day 12 post-CLP (Figure $3 \mathrm{E})$.

Altogether, the numbers of splenic T cells and B cells were reduced through all phases of sepsis. Splenic macrophage numbers were decreased in the early phase of sepsis (day 1 post-CLP), but were increased in the late phase (day 12 postCLP). Splenic macrophages might lose their function, with less activation markers at day 5 post-CLP; however, cell numbers and activation were restored at the late phase of sepsis.
A

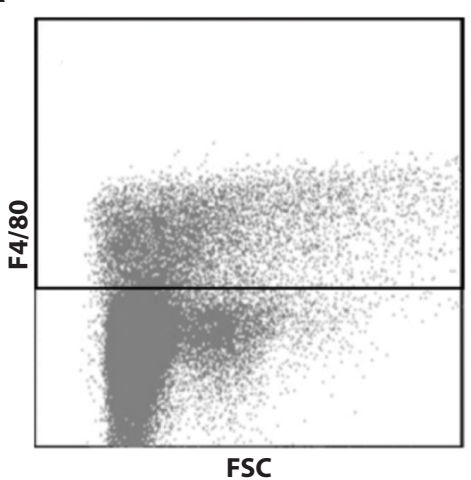

B

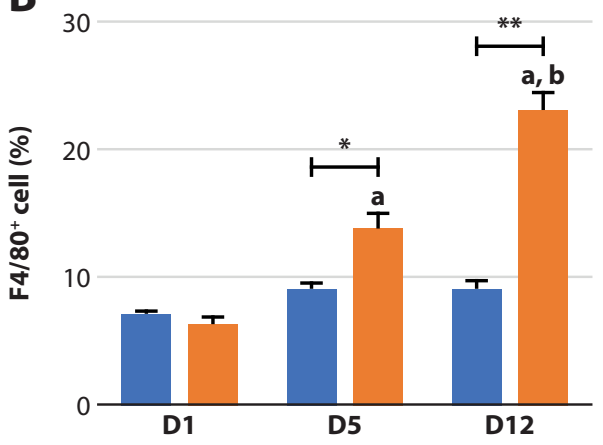

D

30

$\mathrm{F} 4 / 80^{+}$gated

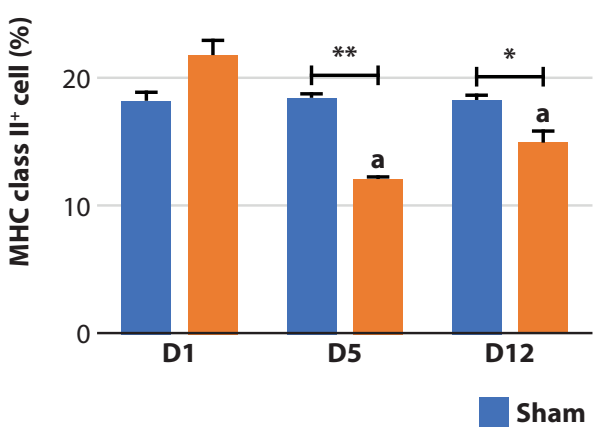

$\mathrm{F} 4 / 80^{+}$gated

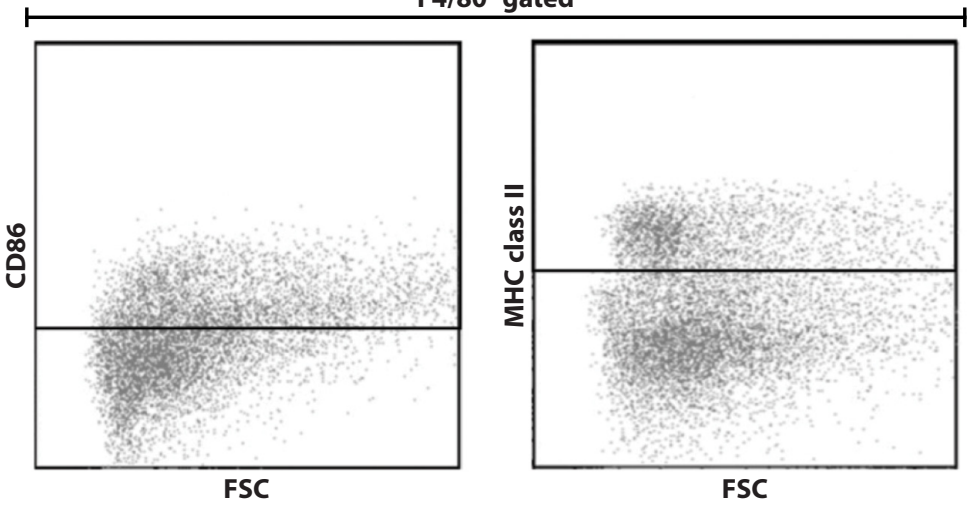

C $\left(\times 10^{6}\right)$
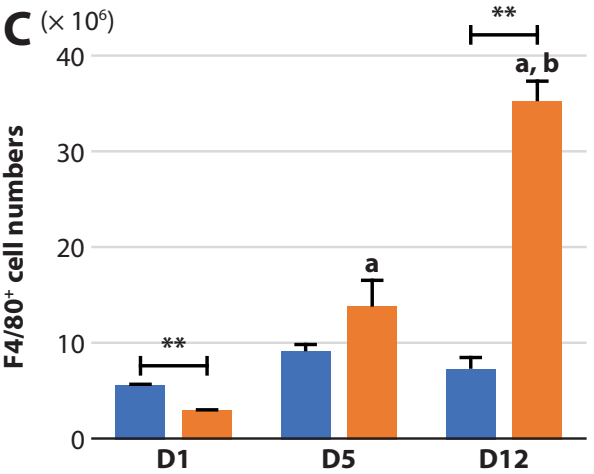

E

40

$\mathrm{F4} / 80^{+}$gated

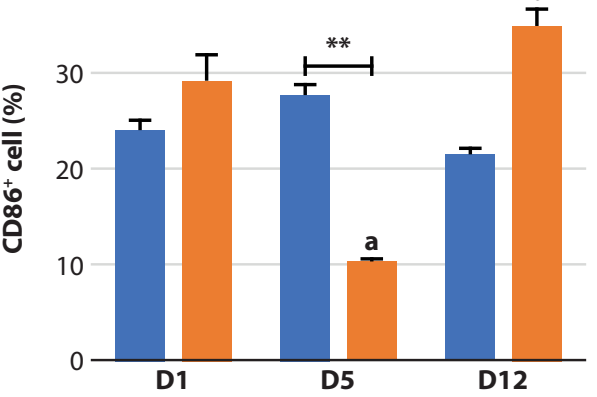

CLP

Figure 3. Changes in macrophage numbers and activation during sepsis.

(A) Dot plot analysis of macrophages. The bulk splenic macrophages were identified by F4/80 marker. The electronic gate was placed on $\mathrm{F} 4 / 80^{+}$population, and the macrophage activation markers, CD86 and MHC class II were analyzed. (B) Percentage of splenic macrophage, $(\mathrm{C})$ numbers of splenic macrophage, (D) percentage of $\mathrm{CD} 86^{+}$macrophages $\left(\mathrm{F} 4 / 80^{+} \mathrm{CD} 86^{+} \mathrm{cells}\right)$, and $(\mathrm{F})$ percentage of MHC class $\mathrm{II}^{+}$macrophages $\left(\mathrm{F} 4 / 80^{+} \mathrm{MHC}\right.$ class $\mathrm{II}^{+}$cells) of CLP and sham control mice were determined at day 1 (D1), day 5 (D5) and day 12 (D12) post-CLP. $n=5$ /group/time point. Data represent two independent experiments. ${ }^{\star} p<0.05$; ${ }^{* *} p<0.01 ;{ }^{a} p<0.05$ vs. D1; ${ }^{b} p<0.05$ vs. D5. 


\section{PD-1 expressing immune phenotype during sepsis}

PD-1 expression was a characteristic marker of immune cell exhaustion in several fatal diseases, including sepsis. ${ }^{1}$ To verify an immune exhaustion state in the CLP mouse model, we investigated the PD-1 expressing immune phenotype in splenic CD4 T cells, CD8 T cells, B cells and macrophages by flow cytometry (Figure 4A). The proportions of $\mathrm{PD}-1^{+}$cells in splenic CD4 T cells $\left(\mathrm{CD}^{+}{ }^{+} \mathrm{CD} 4^{+}\right.$cells) and B cells $\left(\mathrm{B} 220^{+}\right.$cells $)$ from CLP mice were notably increased at day 5 post-CLP, and these increases were sustained until day 12 post-CLP, when compared to the control (Figure 4B, left and right panel). For the splenic $\mathrm{CD} 8 \mathrm{~T}$ cell population $\left(\mathrm{CD} 3^{+} \mathrm{CD} 8^{+}\right.$cells), the high frequency of $\mathrm{PD}-1^{+}$cells was only observed at day 12 post-CLP (Figure 4B, middle panel).
The proportion of $\mathrm{PD}-1^{+}$cells in splenic macrophages $\left(\mathrm{F} 4 / 80^{+}\right.$cells) of mice with sepsis was markedly increased at day 1 post-CLP, and decreased to baseline levels at day 5 and 12 post-CLP (Figure 4C, left panel). Similarly, increased PD$1^{+}$cells were found in $\mathrm{MHC}$ class $\mathrm{II}^{+} \mathrm{F} 480^{+}$macrophages in CLP splenocytes at day 1 post-CLP, and these numbers were decreased on days 5 and 12 post-CLP (Figure 4C, middle panel). A high frequency of $\mathrm{PD}-1^{+}$cells was also found in splenic $\mathrm{CD} 86^{+} \mathrm{F} 480^{+}$macrophages of mice with sepsis on days 1 and 5 post-CLP (Figure 4C, right panel).

Collectively, our data indicated that macrophages and adaptive immune cells exhibited an exhausted phenotype in the early phase (day 1 post-CLP) and late phase (day 5-12 post-CLP) of sepsis, respectively.
A

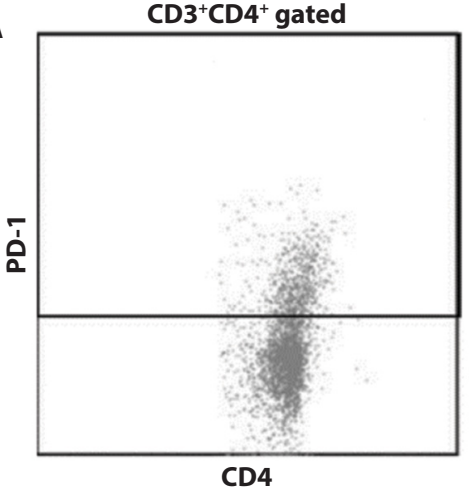

CD4

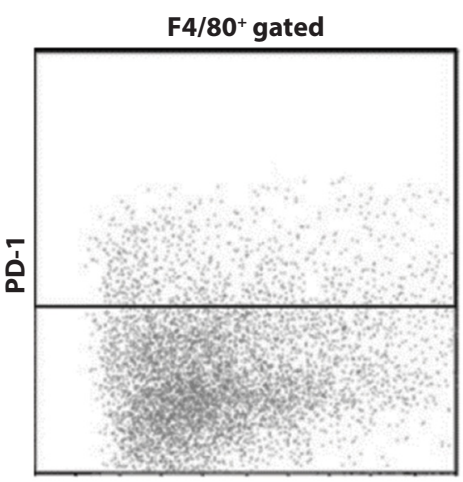

FSC

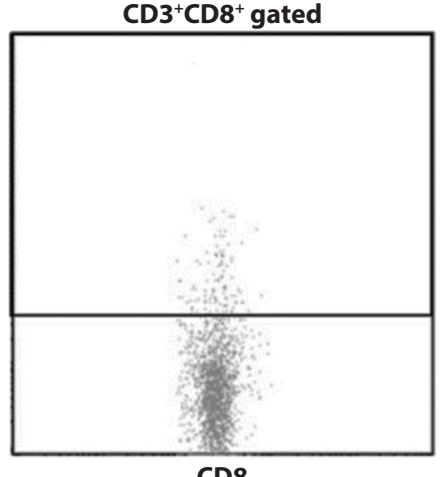

CD8

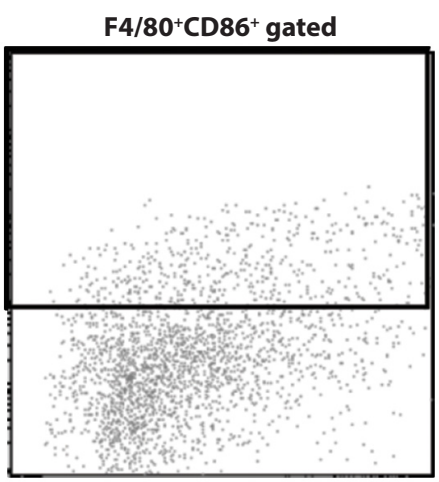

FSC

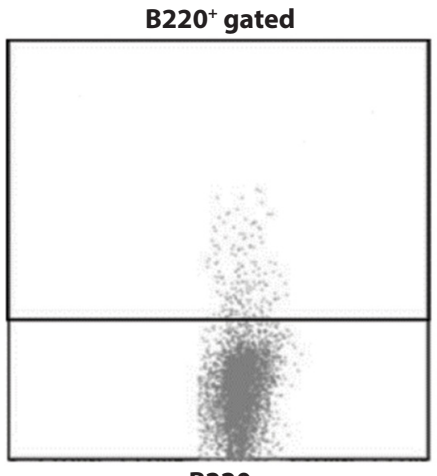

B220

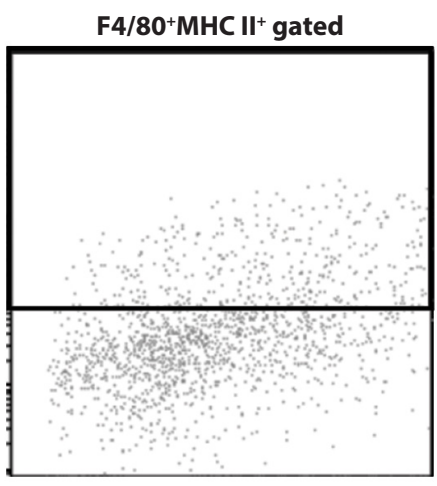

FSC

Figure 4. Kinetic expression of PD-1 molecules on immune cells during sepsis.

(A) Dot plot analysis of PD $-1^{+}$cells. Upper panel, splenic CD4 T cells (CD3 ${ }^{+} \mathrm{CD} 4^{+}$cells), $\mathrm{CD} 8 \mathrm{~T}$ cells $\left(\mathrm{CD} 3^{+} \mathrm{CD} 8^{+}\right.$cells) and $\mathrm{B}$ cells $\left(\mathrm{B} 220^{+}\right.$cells) were identified by the electronic gates based on their lineage markers, and PD- $1^{+}$fractions were subsequently gated in each immune cell subset. Lower panel, splenic macrophages (F4/80 ${ }^{+}$cells), CD86 macrophages (F4/80 ${ }^{+} \mathrm{CD} 86^{+}$cells), and $\mathrm{MHC}$ class $\mathrm{II}^{+}$macrophages $\left(\mathrm{F} 4 / 80^{+} \mathrm{MHC}\right.$ class $\mathrm{II}^{+}$cells) were identified by the electronic gates, and $\mathrm{PD}-1^{+}$fractions were subsequently gated. Percentage of $\mathrm{PD}-1^{+}$cells in (B) $\mathrm{CD}^{+} \mathrm{CD} 4^{+}$cells, (C) $\mathrm{CD}^{+} \mathrm{CD}^{+}$cells, (D) B220 ${ }^{+}$cells, (E) F4/80 ${ }^{+}$cells, (F) $\mathrm{F} 4 / 80^{+} \mathrm{CD} 86^{+}$cells, and (G) F4/80 ${ }^{+} \mathrm{MHC}$ class $\mathrm{II}^{+}$cells from spleens of CLP or sham control mice were examined at day 1 (D1), day 5 (D5) and day 12 (D12) post-CLP. $n=5$ /group/time point. Data represent two independent experiments. ${ }^{*} p<0.05 ;{ }^{* *} p<$ $0.01 ;{ }^{\mathrm{a}} p<0.05$ vs. D1; ${ }^{\mathrm{b}} p<0.05$ vs. D5. 

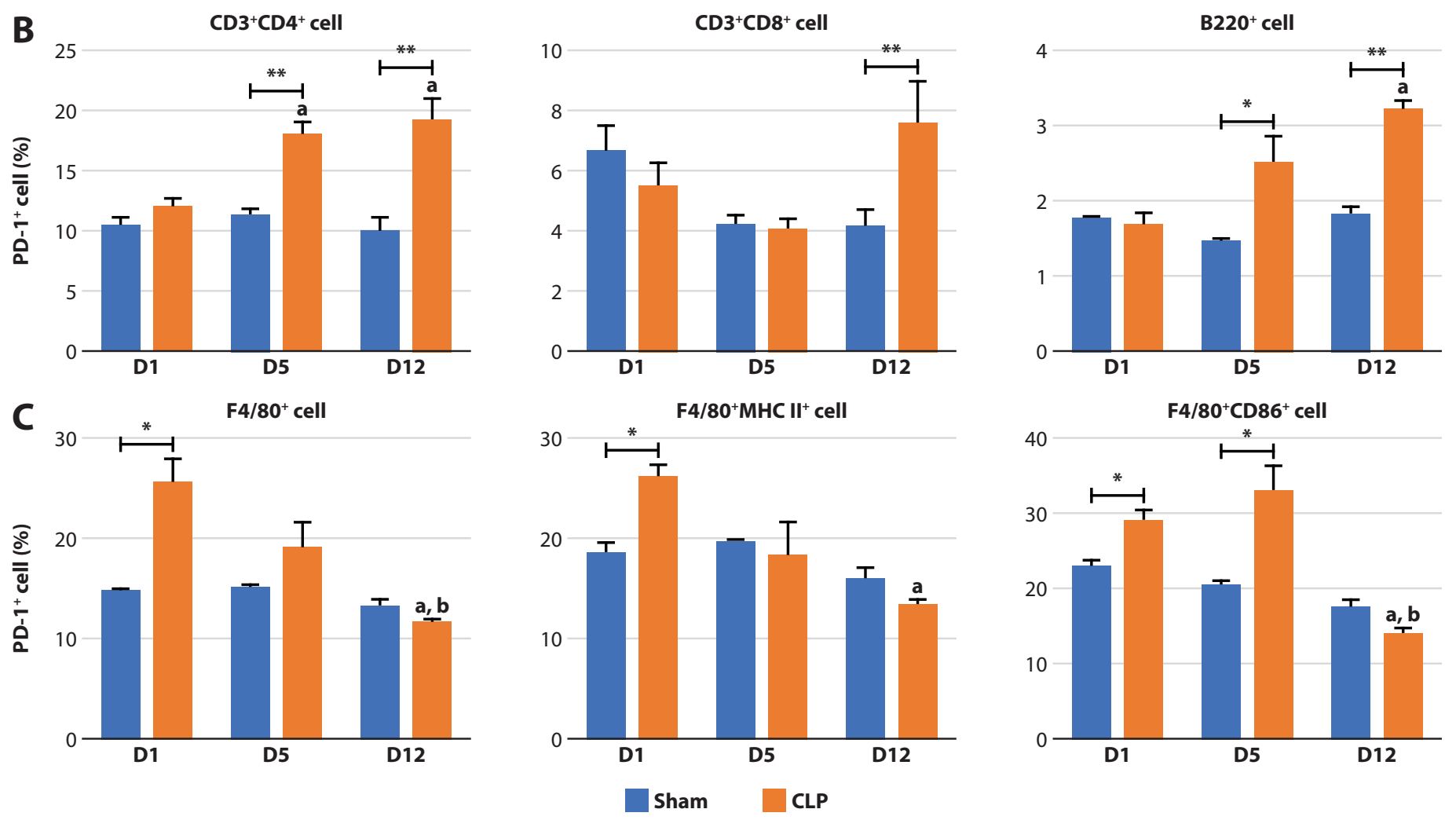

Figure 4. (Continued)

The influence of immune phenotype during sepsis on secondary fungal infection

To evaluate the clinical significance of the immune phenotype during sepsis, CLP and control mice were systemically infected with $C$. albicans on days 5 and 12 post-CLP. These time points were selected based on the changes in macrophage activation (Figure 3), and the alteration of PD-1 expression on macrophages, $T$ cells and $B$ cells (Figure 4). In the CLP group, fungal infection at day 5 post-CLP (CLP D5-Ca)

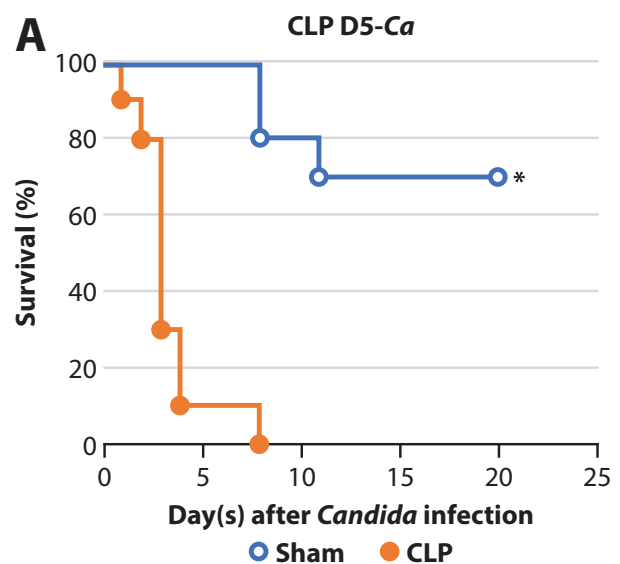

resulted in a mortality rate of $100 \%$ (Figure 5A), while infection at day 12 post-CLP (CLP D12-Ca) produced a $40 \%$ mortality rate (Figure 5B). Consistent with the mortality rate, fungal burden in the blood of the CLP D5-Ca group was high on day 3 post-fungal infection, when compare to those of the infected control mice (Figure 5C). By contrast, fungal burden of the CLP D12-Ca group was comparable to that of the infected control mice (Figure $5 \mathrm{C})$.

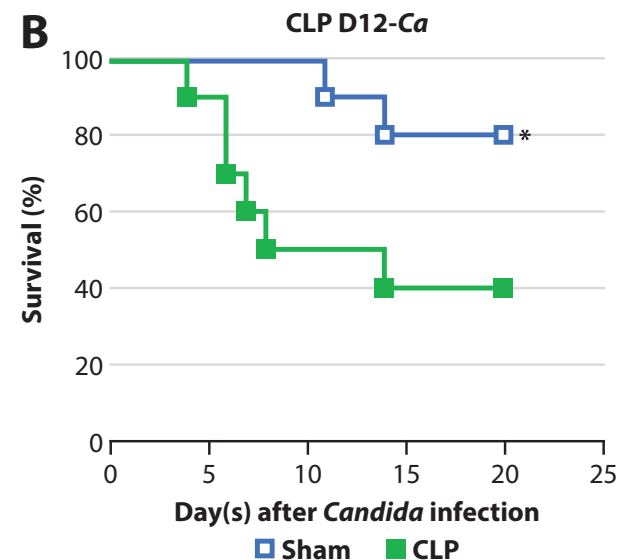

口 Sham CLP

Figure 5. Survival rate and fungal burden of sepsis mice with secondary Candida infection.

Survival analyses of CLP or sham control mice infected with C. albicans at (A) day 5 post-CLP (CLP D5-Ca), and (B) day 12 post-CLP (CLP D12-Ca). (C) Fungal burden in the blood of CLP or sham control mice infected with C. albicans at day 5 postCLP (CLP D5-Ca), and day 12 post-CLP (CLP D12-Ca) were determined after intravenous Candida inoculation at the indicated time points. $n=10 .{ }^{\star} p<0.05 ;{ }^{\dagger} p<0.05$ vs. CLP D12-Ca. 


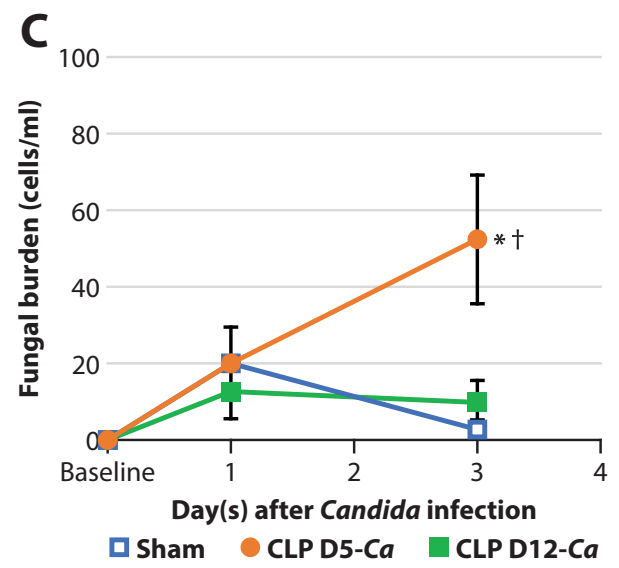

Figure 5. (Continued)

Serum parameters were evaluated to assess organ injury and immune status after secondary fungal infection. At day 3 post-fungal infection, only the CLP D5-Ca group showed a significant increase in serum creatinine and ALT, which indicated acute renal failure and liver injury, respectively (Figure 6A-B). The highest levels of serum TNF- $\gamma$ and IL-6 were also detected in the CLP D5-Ca group (Figure 6C-D), which were related to the organ injury. Although IL-10 was enhanced in the serum of CLP D5-Ca mice (Figure 6E), it did not reduce disease severity or the mortality rate (Figure 5A). IFN- $\gamma$ had been shown to play a pivotal role in anti-fungal immunity. ${ }^{18}$ Therefore, we examined its level in serum following secondary candidiasis. Serum IFN- $\gamma$ levels were significantly lower in both the CLP D5-Ca and CLP D12-Ca groups (Figure 6F), which possibly resulted from reduced $\mathrm{T}$ cell numbers and increased PD-1 expressing $\mathrm{T}$ cells (Figures 3 and 4). Notably, IFN- $\gamma$ levels in the CLP D12-Ca group did not differ from those of the CLP D5-Ca group.
A 0.8

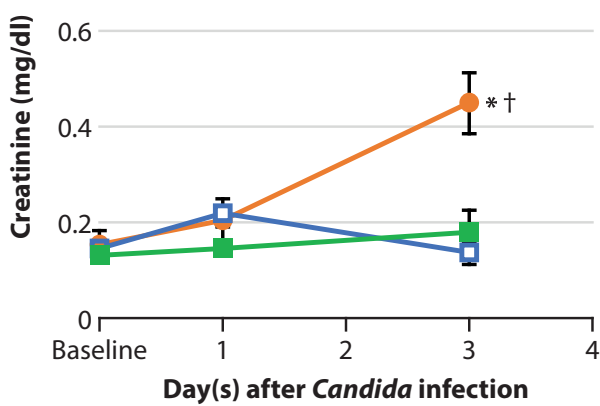

D
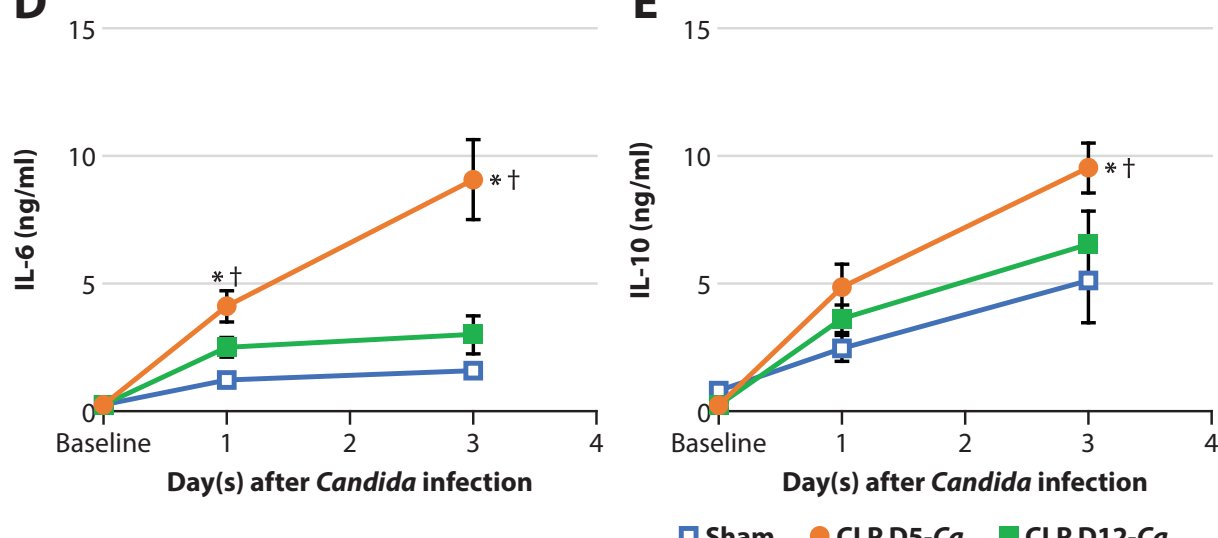

C

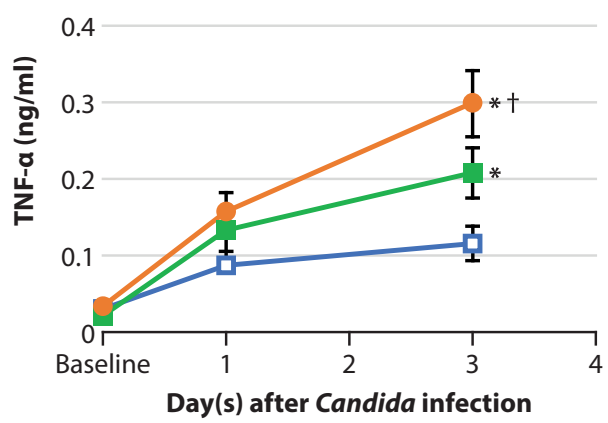

E
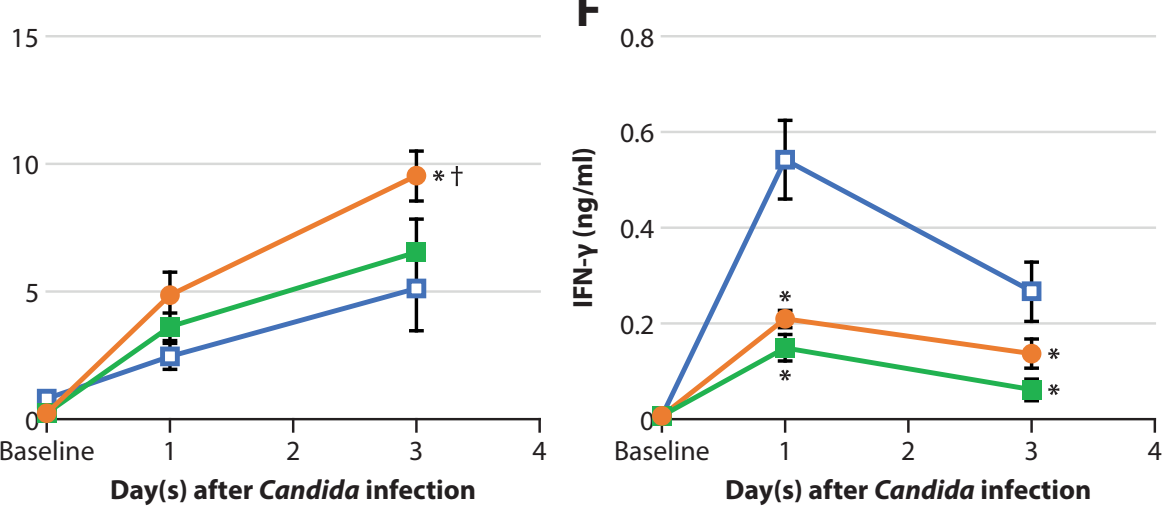

Figure 6. Severity of sepsis mice with secondary Candida infection.

(A) Serum creatinine, (B) serum alanine transaminase (ALT), and (C-F) serum cytokines of CLP or sham control mice infected with $C$. albicans at day 5 post-CLP (CLP D5- $\mathrm{Ca}$ ) were assessed after intravenous Candida inoculation at the indicated time points. $n=10 .{ }^{*} p<0.05 ;{ }^{\dagger} p<0.05$ vs. CLP D12-Ca. 
Our data suggested that mice with sepsis were highly susceptible to secondary Candida infection at day 5 post-CLP, and disease severity might be due to acute organ injury and the hyper-inflammatory reaction. Mice with sepsis developed resistance against secondary Candida infection, with lower inflammatory responses, after day 12 post-CLP.

\section{Discussion}

Secondary fungal infection frequently occurs as a complication of bacterial sepsis due to concomitant immunosuppression. ${ }^{1}$ Our CLP-induced sepsis model exhibited hyper-inflammation (cytokine storm) as early as day 1 post-CLP, and immune exhaustion thereafter, as demonstrated by an increased susceptibility to secondary Candida infection at 5 days post-CLP. CLP mice eventually restored their immunity to overwhelm the fungal infection after 12 days post-CLP.

Hyper-inflammation developed in the early phase ( 1 day post-CLP) of the CLP mouse model, and this response was one underlying mechanism that perpetuated organ injuries (Figure 1). ${ }^{19}$ In the same phase, a reduction in total splenocytes, $\mathrm{T}$ cells, B cells, and macrophages was also observed (Figures 2, 3), and this loss of immune cells was probably the result of inflammation-induced apoptosis. ${ }^{19,20}$ Although an anti-inflammatory cytokine, IL-10, also increased at day 1 postCLP, it was merely a negative feedback of inflammatory regulation to protect against self-damage. ${ }^{21}$

The adaptive immune cells were persistently decreased throughout all phases of sepsis (Figure 2), and cell loss in the later phase of sepsis (5 to 12 days post-CLP) might be, in part, due to immune exhaustion. PD-1 plays a pivotal role in immune suppression owing to its diverse immunoregulatory properties, and an increase and sustained expression of PD-1 is a hallmark of an exhausted immune phenotype in lethal infectious diseases, including sepsis. ${ }^{1,8}$ One well-known function of PD-1 is that it can direct exhausted immune cells to undergo apoptosis. ${ }^{13}$ Therefore, the loss of adaptive immune cells during the late phase of sepsis (day 5 to 12 post-CLP; Figure 2) was probably associated with the upregulation of PD-1 molecules (Figure 4). Although there was sustained exhaustion and reduction of T cells and B cells from day 5 to 12 post-CLP, mice with sepsis only displayed high susceptibility to secondary Candida infection at day 5, and not at day 12 post-CLP.

The number of activated macrophages $\left(\mathrm{F} 4 / 80^{+} \mathrm{CD} 86^{+}\right.$and $\mathrm{F} 4 / 80^{+} \mathrm{MHC}$ class $\mathrm{II}^{+}$cells) at day 5 post-CLP was notably decreased, and about half the $\mathrm{F} 4 / 80^{+} \mathrm{CD} 86^{+}$population expressed PD-1 (Figures 3 and 4). Our results were consistent to previous observations of human and mouse sepsis, which found a decreased number of $\mathrm{CD}^{+} 6^{+}$and $\mathrm{HLA}^{-\mathrm{DR}^{+}}$(or MHC class $\mathrm{II}^{+}$) cells in blood. ${ }^{10,22}$ In addition, substantial PD-1 expression was detected on peritoneal macrophages from mice with sepsis, and this was associated with macrophage dysfunction. ${ }^{23}$ Therefore, the deficit of activated macrophages and their exhausted phenotype at day 5 post-CLP might contribute to the increased susceptibility to secondary Candida infection (Figure 5). By contrast, macrophages in mice with sepsis at day 12 post-CLP were reconstituted, and the increased macrophage numbers were related to an increase in total splenocytes (Figures 1B and 3C). The macrophage reconstitution after sepsis was possibly from hematopoietic stem cell-derived macrophages or self-renewal tissue resident macrophages, as described previously. ${ }^{24}$ A large number of $\mathrm{CD}^{2} 6^{+} \mathrm{F} 4 / 80^{+}$activated macrophages were also observed at day 12 post-CLP, in accordance with decreased PD-1 expression on macrophages (Figure $3 \mathrm{E}$ and $4 \mathrm{C}$ ), and these reinvigorated macrophages might account for the resistance to secondary Candida infection. In this regard, the activation and exhaustion of macrophages perhaps contributed to host defenses against secondary fungal infection during sepsis.

$\mathrm{T}$ helper 1 (Th1) cell responses are crucial for protection against Candida bloodstream infection, and IFN- $\gamma$ is a key cytokine mediating anti-Candida immunity. ${ }^{18}$ Hence, systemic IFN- $\gamma$ production in CLP mice, both pre- and post-fungal infection, was investigated. The level of serum IFN- $\gamma$ from day 5 to 12 post-CLP was inconsistent with the reduced $\mathrm{T}$ cell numbers (Figure 1 and 2). It was likely that IFN- $\gamma$ was not involved in fungal clearance, as consistently low IFN- $\gamma$ production was observed in the CLP D5-Ca and CLP D12$\mathrm{Ca}$ groups after Candida infection (Figure 6). Even so, IFN- $\gamma$ has recently been used as an adjunctive therapy against fungal infection, ${ }^{25,26}$ though treatment with IFN- $\gamma$ alone was not effective. ${ }^{27}$ Although IL-17 has been suggested to play a key role in Candida eradication, its role is restricted to mucosal infections. ${ }^{28}$ Furthermore, our recent investigation demonstrated that IFN- $\gamma$, but not IL-17, was a significant protective cytokine for host defense against secondary fungal infection in murine sepsis. ${ }^{29}$

In summary, the reduced numbers of $\mathrm{T}$ and $\mathrm{B}$ cells during sepsis were possibly related to PD-1 expression; however, these changes were irrelevant to the susceptibility to secondary Candida infection. By contrast, for secondary fungal infection during sepsis, macrophage exhaustion and activation were possibly involved in susceptibility and resistance, respectively. In addition, IFN- $\gamma$ might be a significant protective cytokine, enforcing protective immunity to eradicate Candida infection from the bloodstream. In the last decade, anti-PD-1 therapy has shown high efficacy in promoting immune responses against fungal infection and bacterial sepsis. Blockade of the PD-1 pathway has improved survival in primary and secondary Candida infections through enhanced IFN- $\gamma$ production, and the restoration of antigen-presenting cell function. ${ }^{30}$ In addition, combination immunotherapy of an anti-PD-1 monoclonal antibody (Nivolumab) and recombinant IFN- $\gamma$ was highly effective in improving survival outcomes of patients with extensive abdominal mucormycosis that was unresponsive to conventional therapy. ${ }^{26} \mathrm{~A}$ recent clinical trial has also demonstrated the high efficacy of anti-PD-1 therapy in patients with sepsis-associated immunosuppression, with no apparent adverse immune effects. ${ }^{31}$ The aforementioned evidence revealed that PD-1 was a promising target for immunotherapy of sepsis and fungal infections. 


\section{Conclusion}

This study characterized the kinetic alterations of immune cell populations and immune phenotypes in CLP-induced sepsis, and their association with secondary fungal infection. The findings indicated that an alteration of innate immune phenotypes during sepsis might be reflected in the innate immune activation state and its function, which might be relevant in susceptibility to secondary fungal infections. From a clinical point of view, our results show the need for further studies in patients with sepsis-associated immunosuppression, to ascertain the implication of innate activation and exhaustion markers in secondary fungal infection. This fundamental knowledge would help in the design of therapeutic strategies aimed at restoring immune function in patients with sepsis, for fungal clearance.

\section{Conflict of interests}

This research was conducted in the absence of any financial interests.

\section{Acknowledgements}

This project was supported by Faculty Research Grant DRF 60003, the Faculty of Dentistry, Chulalongkorn University, the Thailand Government Fund (RSA-6080023), the Thailand Research Fund (RES_61_202_30_022), and the Ratchadaphiseksomphot Endowment Fund (76001-HR) at Chulalongkorn University. Research Units were supported by the Ratchadaphiseksomphot Endowment Fund. Chau T. B. V. was supported by The $100^{\text {th }}$ Anniversary Chulalongkorn University Fund for Doctoral Scholarship. We thank the Oral Biology Research Center, Faculty of Dentistry, Chulalongkorn University for the use of research equipment and flow cytometry services.

\section{References}

1. Hotchkiss RS, Monneret G, Payen D. Sepsis-induced immunosuppression: from cellular dysfunctions to immunotherapy. Nat Rev Immunol. 2013;13(12):862-74.

2. Hotchkiss RS, Moldawer LL, Opal SM, Reinhart K, Turnbull IR, Vincent JL. Sepsis and septic shock. Nat Rev Dis Primers. 2016;2:16045.

3. Suleyman G, Alangaden GJ. Nosocomial Fungal Infections: Epidemiology, Infection Control, and Prevention. Infect Dis Clin North Am. 2016;30(4):1023-52.

4. Ostrosky-Zeichner L, Al-Obaidi M. Invasive Fungal Infections in the Intensive Care Unit. Infect Dis Clin North Am. 2017;31(3):475-87.

5. Zhao GJ, Li D, Zhao Q, Song JX, Chen XR, Hong GL, et al. Incidence, risk factors and impact on outcomes of secondary infection in patients with septic shock: an 8-year retrospective study. Sci Rep. 2016;6:38361

6. Taccone FS, Van den Abeele AM, Bulpa P, Misset B, Meersseman W, Cardoso $\mathrm{T}$, et al. Epidemiology of invasive aspergillosis in critically ill patients: clinical presentation, underlying conditions, and outcomes. Crit Care. 2015;19:7

7. Kett DH, Azoulay E, Echeverria PM, Vincent JL, Extended Prevalence of Infection in ICUSGoI. Candida bloodstream infections in intensive care units: analysis of the extended prevalence of infection in intensive care unit study. Crit Care Med. 2011;39(4):665-70.

8. Sharpe AH, Pauken KE. The diverse functions of the PD1 inhibitory pathway. Nat Rev Immunol. 2018;18(3):153-67.

9. Wherry EJ. T cell exhaustion. Nat Immunol. 2011;12(6):492-9.
10. Boomer JS, To K, Chang KC, Takasu O, Osborne DF, Walton AH, et al. Immunosuppression in patients who die of sepsis and multiple organ failure. JAMA. 2011;306(23):2594-605.

11. Guignant C, Lepape A, Huang X, Kherouf H, Denis L, Poitevin F, et al. Programmed death-1 levels correlate with increased mortality, nosocomial infection and immune dysfunctions in septic shock patients. Crit Care. 2011;15(2):R99.

12. Brahmamdam $\mathrm{P}$, Inoue $\mathrm{S}$, Unsinger $\mathrm{J}$, Chang $\mathrm{KC}$, McDunn JE, Hotchkiss RS. Delayed administration of anti-PD-1 antibody reverses immune dysfunction and improves survival during sepsis. J Leukoc Biol. 2010;88(2):233-40.

13. Zhang Y, Zhou Y, Lou J, Li J, Bo L, Zhu K, et al. PD-L1 blockade improves survival in experimental sepsis by inhibiting lymphocyte apoptosis and reversing monocyte dysfunction. Crit Care. 2010;14(6): R220.

14. Hubbard WJ, Choudhry M, Schwacha MG, Kerby JD, Rue LW, $3^{\text {rd }}$, Bland KI, et al. Cecal ligation and puncture. Shock. 2005;24 Suppl 1:52-7.

15. Ertel W, Morrison MH, Ayala A, Dean RE, Chaudry IH. Interferon-gamma attenuates hemorrhage-induced suppression of macrophage and splenocyte functions and decreases susceptibility to sepsis. Surgery. 1992;111(2):177-87.

16. Kumar V. Targeting macrophage immunometabolism: Dawn in the darkness of sepsis. Int Immunopharmacol. 2018;58:173-85.

17. Qiu P, Liu Y, Zhang J. Review: the Role and Mechanisms of Macrophage Autophagy in Sepsis. Inflammation. 2019;42(1):6-19.

18. Davidson L, Netea MG, Kullberg BJ. Patient Susceptibility to Candidiasis-A Potential for Adjunctive Immunotherapy. J Fungi (Basel). 2018;4(1).

19. Bosmann M, Ward PA. The inflammatory response in sepsis. Trends Immunol. 2013;34(3):129-36.

20. Leelahavanichkul A, Yasuda $\mathrm{H}$, Doi K, Hu X, Zhou H, Yuen PS, et al. Methyl-2-acetamidoacrylate, an ethyl pyruvate analog, decreases sepsis-induced acute kidney injury in mice. Am J Physiol Renal Physiol. 2008;295(6):F1825-35.

21. Couper KN, Blount DG, Riley EM. IL-10: the master regulator of immunity to infection. J Immunol. 2008;180(9):5771-7.

22. Nolan A, Kobayashi H, Naveed B, Kelly A, Hoshino Y, Hoshino S, et al. Differential role for CD80 and CD86 in the regulation of the innate immune response in murine polymicrobial sepsis. PLoS One. 2009;4(8):e6600.

23. Huang X, Venet F, Wang YL, Lepape A, Yuan Z, Chen Y, et al. PD-1 expression by macrophages plays a pathologic role in altering microbial clearance and the innate inflammatory response to sepsis. Proc Natl Acad Sci U S A. 2009;106(15):6303-8.

24. Perdiguero EG, Geissmann F. The development and maintenance of resident macrophages. Nat Immunol. 2016;17(1):2-8.

25. Delsing CE, Gresnigt MS, Leentjens J, Preijers F, Frager FA, Kox M, et al. Interferon-gamma as adjunctive immunotherapy for invasive fungal infections: a case series. BMC Infect Dis. 2014;14:166

26. Grimaldi D, Pradier O, Hotchkiss RS, Vincent JL. Nivolumab plus interferon-gamma in the treatment of intractable mucormycosis. Lancet Infect Dis. 2017;17(1):18.

27. El-Khoury M, Ligot R, Mahoney S, Stack CM, Perrone GG, Morton CO. The in vitro effects of interferon-gamma, alone or in combination with amphotericin B, tested against the pathogenic fungi Candida albicans and Aspergillus fumigatus. BMC Res Notes. 2017;10(1):364.

28. Netea MG, Joosten LA, van der Meer JW, Kullberg BJ, van de Veerdonk FL. Immune defence against Candida fungal infections. Nat Rev Immunol. 2015;15(10):630-42.

29. Vu CTB, Thammahong A, Yagita H, Azuma M, Hirankarn N, Ritprajak P, et al. Blockade of PD-1 Attenuated Post-Sepsis Aspergillosis via The Activation Of IFN-gamma and The Dampening of IL-10. Shock. 2019.

30. Chang KC, Burnham CA, Compton SM, Rasche DP, Mazuski RJ, McDonough JS, et al. Blockade of the negative co-stimulatory molecules PD-1 and CTLA-4 improves survival in primary and secondary fungal sepsis. Crit Care. 2013;17(3):R85.

31. Hotchkiss RS, Colston E, Yende S, Angus DC, Moldawer LL, Crouser $\mathrm{ED}$, et al. Immune Checkpoint Inhibition in Sepsis: A Phase 1b Randomized, Placebo-Controlled, Single Ascending Dose Study of Antiprogrammed Cell Death-Ligand 1 Antibody (BMS-936559). Crit Care Med. 2019;47(5):632-42. 